\title{
Composite Paraganglioma
}

National Cancer Institute

\section{Source}

National Cancer Institute. Composite Paraganglioma. NCI Thesaurus. Code C157246.

A neoplasm that combines morphologic characteristics of paragang lioma and

neuroectodermal tumors such as neuroblastoma, ganglioneuroma,

gang lioneuroblastoma, or peripheral nerve sheath tumor. 